\title{
Experimental investigation of the influences of different liquid types on acoustic emission energy levels during the bubble formation process
}

\author{
Taihiret Alhashan ${ }^{1}$ - Abdulmajid Addali ${ }^{1}$ Joao Amaral Teixeira ${ }^{1}$. \\ Abdelhamid Naid ${ }^{2}$
}

Received: 3 April 2017/Accepted: 31 August 2017/Published online: 8 September 2017

(c) The Author(s) 2017. This article is an open access publication

\begin{abstract}
Bubble formation phenomena in a two-phase gas/liquid system occur in many industries that involve boiling; such as desalination stations, nuclear reactors, chemical plants, and fluid piping transportation and processes. Bubble formation phenomena cause problems, such as a decrease in equipment efficiency, vibration, noise, and solid surface erosion. Applications of the acoustic emission (AE) technique for monitoring bubble formation and burst stages in boiling processes are marginal in terms of extension in comparison to other applications of the $\mathrm{AE}$ technique. The use of the $\mathrm{AE}$ technique in this experimental investigation covers the frequency range between 100 and $1000 \mathrm{kHz}$, showing that the AE sensor can detect acoustic emissions from an occurrence of bubble formation. Statistically, it was found that the best AE parameter indicator for bubble formation was AE-RMS (root mean square).
\end{abstract}

Keywords Acoustic emission - Bubble formation · Pool boiling vessel

\author{
List of symbols \\ $f_{0} \quad$ Frequency of the bubble \\ $d$ Bubble diameter \\ $\gamma \quad$ Polytrophic constant of the gas inside the bubble \\ $P_{0} \quad$ Hydrostatic pressure \\ $\rho \quad$ Liquid density
}

Taihiret Alhashan

t.a.alhashan@cranfield.ac.uk

1 School of Energy, Cranfield University, Cranfield, Beds MK43 0AL, UK

2 School of Mechanical Engineering, Azzaytuna UniversityLibya, Bani Walid, Libya

\section{Introduction}

Bubble formation and cavitation are common phenomena in many industrial processes that include gas-liquid systems or just liquid flow, such as manufacturing processes, which use gas or water jets that are blown into a liquid to provide energising processes, and in gas-liquid flows that commonly occur in chemical processing plants. However, while cavitation may not affect the surface integrity of a liquid, the collapse of cavitation bubbles close to the wall or other surfaces can cause active surface erosion, known as cavitation damage. In liquid transportation industries, the cavitation phenomenon is a contributor to the failure and inefficiency of pumps and valves that leads to a decrease in the life of the equipment [1]. For liquid power systems, such as in gas-lift pumps, cavitation will cause vibration, noise, reduced efficiency, pitting, and erosion of the surfaces in contact with collapsing bubbles [2]. Weninger et al. [3] noted that the bubble collapse generates an amount of energy in the form of a shockwave and that transducers can measure its pressure pulse. It is possible to correlate pressure pulses from bubble activity with bubble size; in other words, through measuring the size of the bubble using an acoustic technique. It is well known that bubble activities are potential sources of $\mathrm{AE}$, and that the acoustic energy released from the bubble formation can be used to determine certain properties of the surrounding liquid [4]. This paper starts with the definition of cavitation, which is followed by a general presentation of bubble occurrence phenomena, and of the acoustic techniques that are suitable for monitoring and detection of bubble formation at the early stages of the boiling processes.

Cavitation is a phenomenon that occurs in rotating machines and flow process, which is characterised by the 
collapse of voids in the flow with an explosive effect generating localised high pressures and temperatures. Cavitation occurs when the liquid is subjected to a drop in pressure, which is equal or lower than the saturated vapour pressure at a given temperature $[5,6]$. To reduce the damage caused by cavitation, bubble formation must be monitored and detected in rotating machines and valves [7]. Acoustic emission is a physical phenomenon that occurs within or on the surface of the material. It is defined as elastic waves generated by the rapid release of energy from localised sources within a material due to a deformation or fracture process [8, 9]. Application of the acoustic emission technique is not limited to detection and diagnosis of cavitation in hydraulic systems, such as centrifugal pumps and valves; it can be used as a monitoring tool for different types of industries, such as petroleum engineering using gas-liquid pumps, mechanical processes, and chemical processing plants. Furthermore, the $\mathrm{AE}$ technique can be applied to the monitoring and diagnosis of bubble formation in boiling processes.

Alhashan et al. [10] used the AE technique in the monitoring of bubble formation during the boiling process. They found a clear association between increasing $\mathrm{AE}$ levels and bubble formation. Benes and Uher [11] found that the parameters of the AE signal have a correlation with overheating during heat transfer. It was established that the AE signal could be used to predict the boiling phenomena. Carmi et al. [12] used the AE technique in a flow boiling experiment to detect bubble transit, noting the possibility of using $\mathrm{AE}$ in the detection of bubble dynamic events at the early stages in the boiling process. The AE technique has been used to diagnose the bubble formation process and monitor bubble departure from the heating surface of the boiler to the surface of the liquid container during pool boiling [13]. In another investigation, centring on twophase flows, Addali [14] found that the gas void fraction (GVF) can be determined by measurement of the acoustic emission. Moreover, it was concluded that there is a direct correlation between the AE energy and GVF. It was also noted that an increase in superficial gas and liquid velocities caused an increase in AE energy levels. Husin et al. [15] found that $\mathrm{AE}$ was a suitable technique for the detection of a single bubble formation and burst, with $\mathrm{AE}$ being used to measure the velocity of the acoustic wave. Jaubert et al. [16] used AE as the method for the detection of cavitation phenomena in pumps and valves at an early stage, which makes it possible to study incipient cavitation occurrences. Husin [17] found that when the bubble size and liquid viscosity increase, the level of $\mathrm{AE}$ signals also increases. One particular item of interest in this programme was the investigation of AE energy radiated from the bubble formation. The bubble formation phenomena have potential energy that depends on the pressure within the bubbles and their volume.

Leighton et al. [18] found that some of the sound emitted from running brooks was caused by the transient pressure waves generated by gas bubble pulsation inside the liquid. Earlier, Minnaert [19] found that the pressure waves were produced from volumetric bubble oscillations during bubble formation. The bubbles are subjected to initial oscillations, which create an acoustic pressure wave before reaching an equilibrium state. Pumphrey and Crum [20] studied the impact of water drops on a free surface using a high-speed camera and found that the majority of the acoustics produced were due to volume pulsations of the bubble, which generate sound. Leighton [21] concluded that the sources of sound emitted from oceans were caused by pressure waves produced by gas bubbles inside the liquid. Strasberg [22] found that the sound occurs only when the bubble is in volume pulsation; he also found that smaller bubbles, with a smaller surface area, radiate less energy for a given volume rate of bubble formation. Bubble formation and bubble collapse generate pressure waves that can be detected within a wide frequency band. Moreover, bubble size can be calculated using Eq. (1) as below, created by Minneart [19], and known as the natural frequency of oscillation of the bubble,

$f_{0}=\frac{1}{\pi d} \sqrt{\frac{3 \gamma P_{0}}{\rho}}$.

The sound created by bubble formation, oscillation and burst at the free surface is dependent on the bubble size. The period of stress pulse due to bubble collapse and burst is very short (ms) [23]. Acoustic emission of bubble burst is correlated to the size of the bubble [15]. For pool boiling, the bubble dynamics are divided into five stages: (1) bubble formation at the bottom of the boiler wall, (2) bubble rising, (3) bubble coalescence, (4) splitting of bubbles, and (5) bubble burst at the free surface [4] [24]. When the bubble cavities collapse, it causes sound and dissipation of energy in the water [25]. It is known that the pressure pulses associated with the bubble formation and burst at the surface of the liquid also act as sources of acoustic emission [26] [27]. Blanchard and Woodcock [28] observed that the bubbles coalesce in tap water more than in sea water. Additionally, the bubbles produced in sea water are smaller and continue longer than in pure water. Ceccio et al. [29] concluded that there is a significant difference between bubble occurrence in salt water and fresh water. It was observed that the acoustic emission of the bubble cavitation in fresh water was lower than that produced in salt water. Moreover, they noted that small bubbles produce higher acoustic emission compared to large bubbles. 
Bubble formation phenomena are considered one of the most common causes of failure in process systems. Centrifugal pumps and valves commonly fail as a result of pitting due to undetected bubble activities including bubble formation and collapse. Thus, monitoring for early detection of bubble formation in hydraulic components is necessary. Successfully implementing a condition monitoring programme that can pick up early signs of bubble formation could lead to operating the process without having to stop the machine for frequent inspections. To date, most previous works showed that there had been few attempts to apply the AE technique for the monitoring and detection of bubble formation processes. There are potentially unlimited possibilities to develop techniques, tools, and applications for effective diagnostic systems. The aim of this research is to investigate the effect of liquid properties on the bubble formation characteristics, such as bubble size, bubble generation rate, detachment of bubble, etc. In fact, the boiling process offers a good opportunity to study bubble formation due to the increase in liquid temperature. AE energy levels are used as a reference parameter to assess the process. Thus, the aimed objectives of this work are:

- Use of AE to monitor the process.

- Study of the effect of different liquid types (tap water, demoralise water, and salt water) on AE signals.

- Investigation of the influence of the input power-supply (2.5-3 kW) on AE levels.

- Study of the effect of threshold levels on AE signals.

\section{Experimental apparatus}

This investigation was built on work previously carried out in this area. In these experiments, a deep vessel was used to separate the bubble formation and bursting regions. Furthermore, three different liquid types and three different liquid levels were used during pool boiling tests, as shown in Table 1.

The boiling tests were performed using a general purpose test-rig, as shown in Fig. 1. It consisted of a water boiler with a $270 \mathrm{~mm}$ inner diameter and $440 \mathrm{~mm}$ in height. The maximum capacity of the boiler is 26 litres. The pool boiler is made of stainless-steel and is integrated with a heater, located at the bottom of the container, to heat the water inside the boiler. The round heater has an external diameter of $150 \mathrm{~mm}$. A constant electrical power of $2500 \mathrm{~W}$ is supplied to the boiler heater throughout the experiments.

A commercially available piezoelectric AE sensor (Physical Acoustic Corporation type "PICO") with an operating range of $100-1000 \mathrm{kHz}$ was used. Two acoustic sensors were attached to the external surface of the boiler using superglue. The two AE channels were distanced at $200 \mathrm{~mm}$, vertically apart. The first channel was connected to the bottom of the boiler, $100 \mathrm{~mm}$ from the bottom surface, to detect the initiation of bubble formation, while channel 2 was positioned $200 \mathrm{~mm}$ above channel 1 to monitor bubble burst, and oscillations when the bubbles depart towards the surface at high water levels (see Fig. 1). The acoustic sensors were linked to a data acquisition system by a preamplifier, set at $40 \mathrm{~dB}$ gain. The system was continuously set to acquire $\mathrm{AE}$ waveforms at a sampling rate of $2 \mathrm{MHz}$. The software (signal processing package "AEWIN") was incorporated within a PC to monitor AE parameters, such as AE-RMS and AE-Energy (recorded at a time constant of $10 \mathrm{~ms}$ and a sampling rate of $100 \mathrm{~Hz}$ ).

Common AE parameters, RMS and Counts, were calculated over a threshold of $36 \mathrm{~dB}$. The threshold value was set at $3 \mathrm{~dB}$ above operational background noise, as shown in Fig. 2, where AE counts over a range of five threshold levels were plotted. To ensure a consistent boiling process throughout the test periods, the measurements of the water temperature were continuously undertaken every second. A thermocouple was fixed inside the pool boiling vessel $3 \mathrm{~mm}$ above the surface of the boiler heater.

\section{Results, observations, and discussions}

\section{Observation of the effect of salt water $(5 \mathrm{~g} / \mathrm{L})$ concentration on AE signals}

Tests were undertaken at three different levels of salt water: 100, 200 and $350 \mathrm{~mm}$. One experimental case is presented, with tests undertaken three times for each of the three levels. The case is a salt water level of $200 \mathrm{~mm}$. Tests were terminated once the water temperature reached $100{ }^{\circ} \mathrm{C}$ (boiling temperature). The start water temperature condition for all tests was $20^{\circ} \mathrm{C}$. Figure 3 below shows the relationship between AE-RMS (V), time (sec) and temperature $\left({ }^{\circ} \mathrm{C}\right)$ at the salt water $(5 \mathrm{~g} / \mathrm{L})$ level of $200 \mathrm{~mm}$.

Figure 3: Observation of boiling test at salt water $(5 \mathrm{~g} /$ L) level of $200 \mathrm{~mm}$.

At the beginning of the process, there was initial bubble formation on the bottom wall of the pool boiling vessel.

Table 1 Experimental procedures of boiler test

\begin{tabular}{ll}
\hline Water type & Water Level $(\mathrm{mm})$ \\
\hline Tap water & $100-200-350$ \\
Demineralized water & \\
Salt water $(5 \mathrm{~g} / \mathrm{L})$ concentration & \\
\hline
\end{tabular}


Fig. 1 Arrangement of the experiment for pool boiling

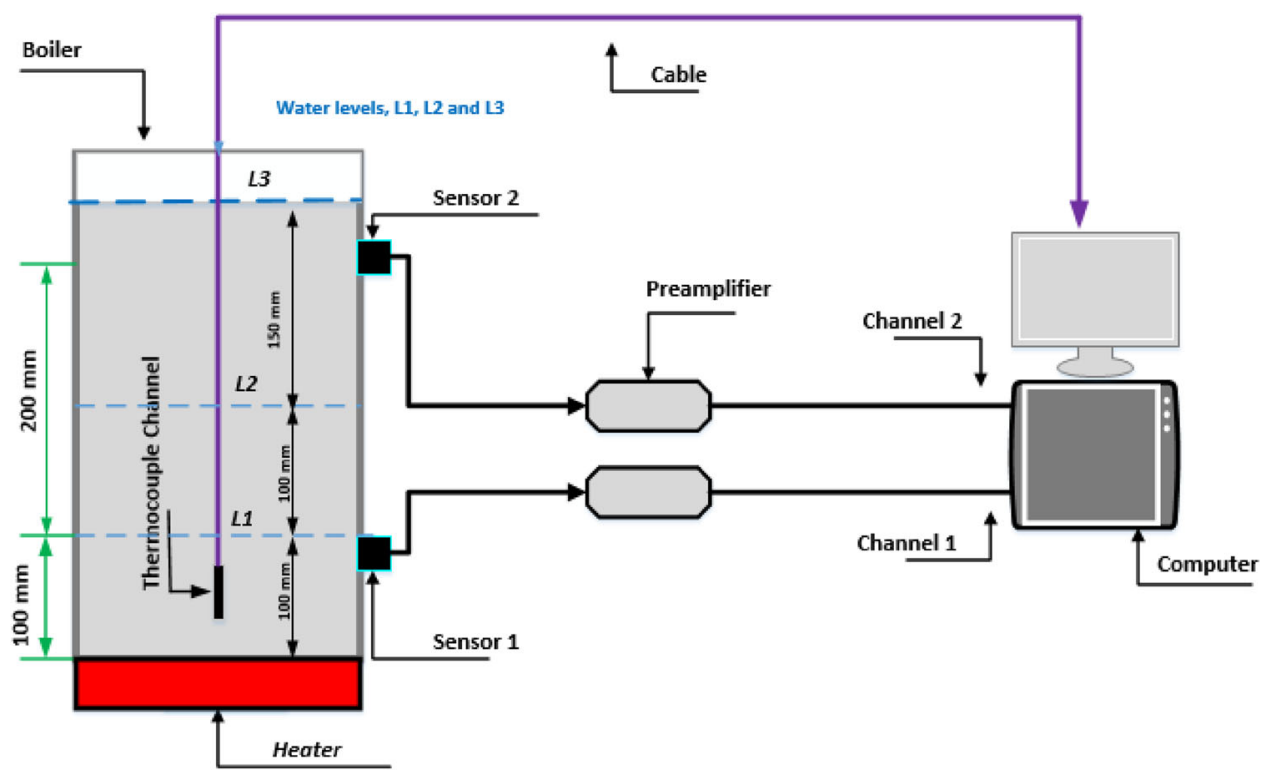

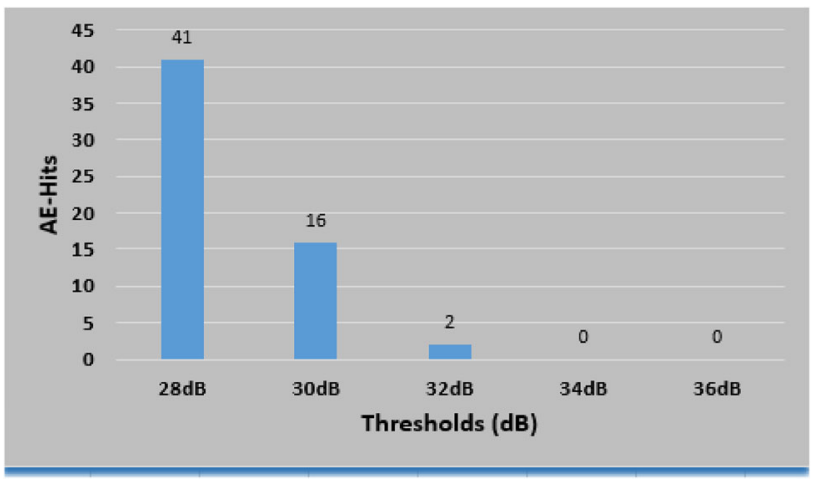

Fig. 2 Threshold levels' test of operation background noise

Moreover, the bubbles formed on cavity, scratches and grooves on the heated surface of the boiler. This process is called heterogeneous nucleation. Then the bubbles detach from the heating surface and rise to the surface of the water; at an early stage at about $22{ }^{\circ} \mathrm{C}$. Bubbling happens on the boundary between the salt water $(5 \mathrm{~g} / \mathrm{L})$ and the solid wall of the boiler. In addition, the bubbles also occur between the water and small parts of salt suspended in the water. For this reason, the bubbles detach from the heating element at an early stage.

The value of AE-RMS ranked the highest for both channels, between 495 and 825 s. During this stage, the temperature rises and the bubbles start to form at a faster rate. Then the bubbles grow and depart towards the free surface, where they burst and release the vapour. As a result, the heat flux attains its maximum value. However, the value of AE-RMS in channel 1 is higher than that of channel 2 , with 0.0009 volts and 0.0005 volts, respectively, because the position of channel 2 is further from the water

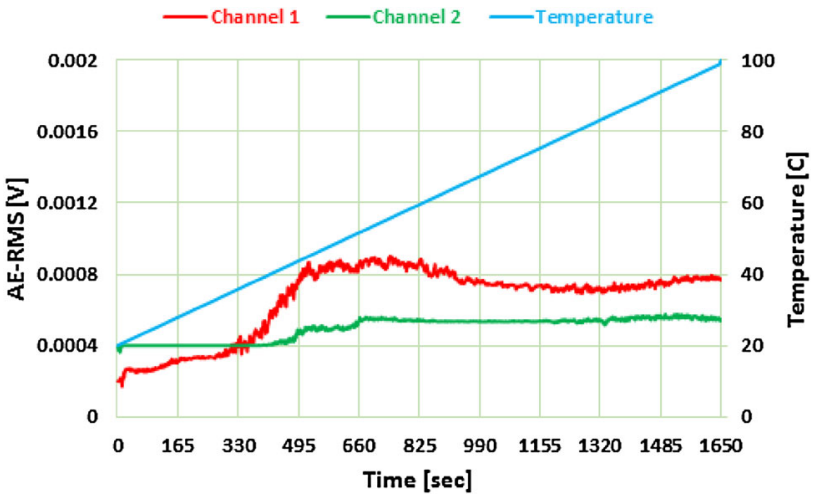

Fig. 3 Observation of boiling test at salt water $(5 \mathrm{~g} / \mathrm{L})$ level of $200 \mathrm{~mm}$

surface. The value of $\mathrm{AE}$ signal decreases slowly to approximately 0.0007 volts in channel 1 , between 60 and $75^{\circ} \mathrm{C}$. During this period, the bubbles start to detach from the heating surface into the surrounding water, some of which rise from the heating wall of the boiler to the water surface, while others combine with adjacent bubbles and then stick to the internal wall of the boiler, causing an isolated layer. This layer is responsible for attenuation of AE signals. Obviously, there is a constant in the value of AE signal for channel 2 , between 55 and $95^{\circ} \mathrm{C}$, because the position of channel 2 is further from the water surface. It was noted that when the salt water $(5 \mathrm{~g} / \mathrm{L})$ approaches the boiling stage, between 90 and $100{ }^{\circ} \mathrm{C}$, the value of $\mathrm{AE}$ increases in both channels. These temperatures cause a rise in the heat flux and more bubbles form and increase whilst travelling towards the free surface, where large bubbles start to burst on the water surface at the end of the test. Also, the size and internal energy of the bubbles increased. 


\section{Observation of the difference between bubble formation in tap water and demineralized water}

Figure 4 below shows the trend of AE signals caused by tap water and demineralized water. In the tap water, the bubbles start to detach from the bottom wall of the boiler at approximately $245 \mathrm{~s}$, and between 30 and $50{ }^{\circ} \mathrm{C}$. During this stage, gas voids appear between the tap water and the small particles suspended in the liquid. It was observed that the level of AE signal decreases gradually between 660 and 1155 s. During this period, the bubbles start to detach from the bottom wall of the pool boiling vessel into the surrounding water. Some of the bubbles then rise from the heating element to the water surface while others combine with adjacent bubbles and stick to the internal wall of the boiler, which causes attenuation of AE signals. The same behaviour happens with the demineralized water but with a different time period. As the water approaches the boiling stage, between 83 and $100{ }^{\circ} \mathrm{C}$, there is a gradual increase in the level of AE signal from 0.0008 volts to approximately 0.0022 volts, as shown in Fig. 4. This growth can be attributed to the heat that was increasing in the water where there was more bubble activity. This heat produced more bubbles to form and move to the top of the water surface where the bubbles' size and their internal energy increased. Finally, these large bubbles started to burst on the water surface at the end of the test.

For the demineralized water, the bubbles start to detach from the heating surface of the boiler at approximately $570 \mathrm{~s}$, between 50 and $60{ }^{\circ} \mathrm{C}$. In this type of water, there are considerably fewer small particles in the liquid from heterogeneous nucleation can occur between the liquid and small particles suspended in the liquid. Furthermore, the thermal motions inside the demineralized water form temporary, microscopic gaps which by themselves can produce nuclei cavitation, which is necessary for the rupture and growth of micro-bubbles. This phenomenon is called homogeneous nucleation.

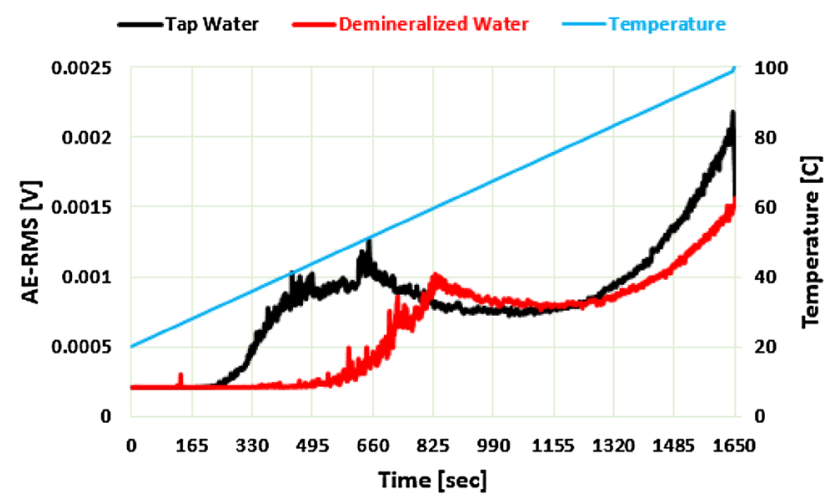

Fig. 4 Correlation between tap water and demineralized water at the water level of $200 \mathrm{~mm}$ (channel 1)
It can be seen that the detachment of bubbles from the heating surface in the tap water is faster than that of demineralized water because there is a much lower concentration of mineral ions and small particles inside the water.

\section{Observation of the difference between bubble formation in tap water and salt water $(5 \mathrm{~g} / \mathrm{L})$}

Figure 5 below shows the relationship between AE-RMS (V), time (s) and temperature $\left({ }^{\circ} \mathrm{C}\right.$ ) for channel 1 , where the black line presents tap water and the green line shows the salt water (5 g/L).

For the tap water, the bubbles start to form on the heating element of the boiler where these bubbles were stuck on the bottom surface of the vessel, which is between 10 and 245 s. At 245 s, the bubbles start to detach from the bottom surface of the boiler into the surrounding water. A sharp transition in the AE movement occurs in tap water, as shown in Fig. 5. This dramatic change indicates the start of the boiling process during pool boiling. More bubbles are formed and depart towards the surface between 30 and $50{ }^{\circ} \mathrm{C}$, due to the heat transferring from the boiler's heating element to the water as the temperature increases. In the salt water $(5 \mathrm{~g} / \mathrm{L})$, the bubbles start to detach from the heating wall of the vessel into the surrounding water at an early stage, between 22 and $45^{\circ} \mathrm{C}$. It was detected that the rate average of bubble detachment from the heating surface in salt water $(5 \mathrm{~g} / \mathrm{L})$ is faster than that of tap water. This is due to the surface tension of salt water $(5 \mathrm{~g} / \mathrm{L})$ being higher than that of tap water. Additionally, bubble gaps appear between the water and small parts of salt suspended in the water, which is responsible for the rupture and growth of micro-bubbles.

In the tap water, many large bubbles appeared and disappeared in a short time. However, there are no large individual bubbles in the salt water. The mechanism of bubble formation in tap water is the same as in salt water

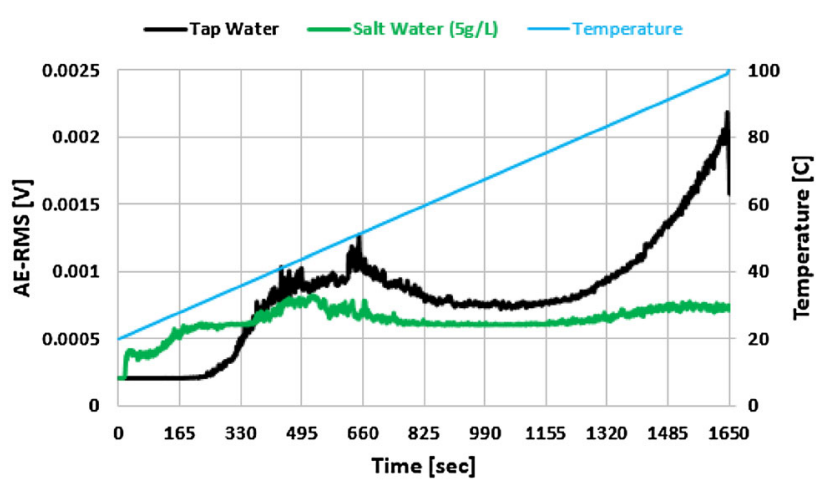

Fig. 5 Correlation between tap water and salt water $(5 \mathrm{~g} / \mathrm{L})$ at the water level of $200 \mathrm{~mm}$ (channel 1) 
but there is a difference in the size of the bubbles. Moreover, the acoustic emission of the bubble formation in tap water was less than that of salt water, between 10 and 330 s, as shown in Fig. 5. Overall, it can be concluded that the bubbles form and rise to the water surface between 30 and $50{ }^{\circ} \mathrm{C}$ in tap water, and between 22 and $45^{\circ} \mathrm{C}$ in salt water.

\section{Observation of the difference between bubble formation in demineralized water and salt water $(5 \mathrm{~g} / \mathrm{L})$}

Figure 6 below shows the relationship between AE-RMS $(\mathrm{V})$, time (s) and temperature $\left({ }^{\circ} \mathrm{C}\right)$ for channel 1 , where the red line presents demineralized water and the green line shows salt water $(5 \mathrm{~g} / \mathrm{L})$.

For demineralized water, there is a stable AE signal from 0 to around $660 \mathrm{~s}$ because the bubbles start to form on the bottom surface of the vessel and then stick on the heating surface between 20 and $45^{\circ} \mathrm{C}$. At around $660 \mathrm{~s}$, the bubbles begin to detach from the boiler's heating wall and rise to the water surface between 50 and $60{ }^{\circ} \mathrm{C}$. This is because the thermal motions inside the liquid create temporary, microscopic gaps that can produce nuclei cavitation, which is necessary for the rupture and growth of micro-bubbles. This process is called homogeneous nucleation. In addition, the value of the AE significantly increases to reach 0.0014 volts, when more bubbles form. The value of the AE signal decreases gradually to approximately 0.00075 volts, between 60 and $78^{\circ} \mathrm{C}$. During this stage, when the bubbles form due to increasing temperature, some of which rise from the heating boiler wall into the surrounding water, others combine with adjacent bubbles and stick to the internal wall of the vessel, causing an isolated layer. This layer is responsible for attenuation of $\mathrm{AE}$ signals. When the water approaches the boiling phase, between 82 and $100{ }^{\circ} \mathrm{C}$, a slight increase in the value of $\mathrm{AE}$ signal from 0.00075 volts to approximately 0.0015 volts in demineralized water was observed, as

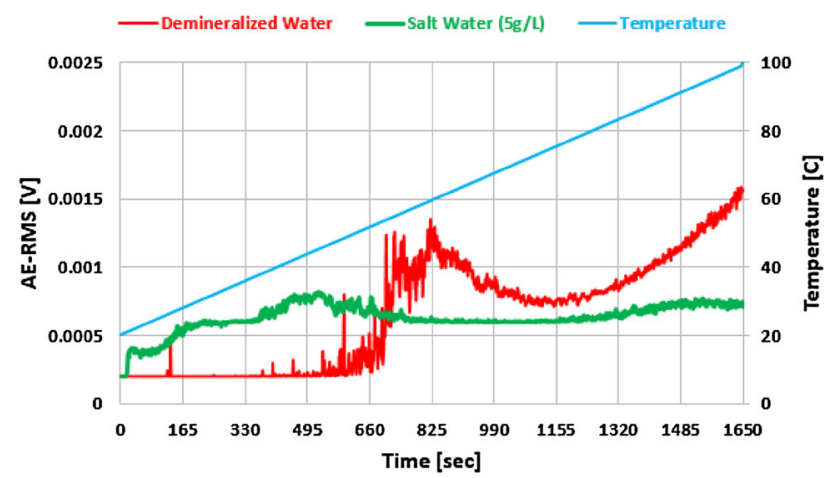

Fig. 6 Correlation between demineralized water and salt water $(5 \mathrm{~g} /$ L) at the water level of $200 \mathrm{~mm}$ (channel 1) shown in Fig. 6. This rise can be attributed to the heat gained by the water. This heat caused more bubbles to form and depart towards the water surface, where finally large bubbles burst on the water surface at the end of the test; and when the bubbles' size and their internal energy increased.

For salt water $(5 \mathrm{~g} / \mathrm{L})$, the bubbles start to detach from the heating element at $22{ }^{\circ} \mathrm{C}$, where more bubbles form and depart from the bottom of the boiler into the surrounding water. During this stage, gas voids occur on the boundary between the liquid and small parts of salt inside the liquid, being suspended in the liquid. For this reason, the bubbles are detached at an early stage from the heating element in the salt water $(5 \mathrm{~g} / \mathrm{L})$. These bubbles combine with each other and then start to split to produce more bubbles.

It can be seen that bubbles detaching from heating surface travelling towards the water surface in the salt water $(5 \mathrm{~g} / \mathrm{L})$ are faster than that of demineralized water. This is because there are small particles of salt within the water that can produce nuclei cavitation, which is necessary for the rupture and growth of micro-bubbles. This event is called hydrophilic surface.

\section{Influence of threshold levels on AE signals}

Figure 7 below shows there is a correlation between AEhits and water levels. In other words, when the liquid level increases, the number of hits also increases due to the increased number of bubbles being formed.

The value of AE-hits was ranked the highest with 160,000 hits for a water level of $350 \mathrm{~mm}$, at $36 \mathrm{~dB}$. Furthermore, the threshold parameters have an effect on $\mathrm{AE}$ signal levels. When the threshold levels increase, the number of hits decreases, as shown in Fig. 7.

\section{Influence of power-supply on AE signals}

Figure 8 shows the relationship between AE-RMS (V) and temperature $\left({ }^{\circ} \mathrm{C}\right)$, where the $x$-axis represents temperature $\left({ }^{\circ} \mathrm{C}\right)$ and the $y$-axis shows the AE signal. The red line shows power-supply at $3 \mathrm{~kW}$ and the black line represents the power-supply at $2.5 \mathrm{~kW}$.

For a power-supply of $2.5 \mathrm{~kW}$, the bubbles start to detach from the heating surface into the surrounding water at around $33{ }^{\circ} \mathrm{C}$. The levels of $\mathrm{AE}$ signal increase between 33 and $50{ }^{\circ} \mathrm{C}$, where more bubbles form caused by heat flux increases. With a power-supply of $3 \mathrm{~kW}$, the bubbles start to detach from the boiler wall bottom travelling towards the water surface directly, at around $22{ }^{\circ} \mathrm{C}$. There is a significant increase in the level of $\mathrm{AE}$ signals between 22 and $50{ }^{\circ} \mathrm{C}$. The increase in the power supply causes a high temperature and heat flux during pool boiling. 


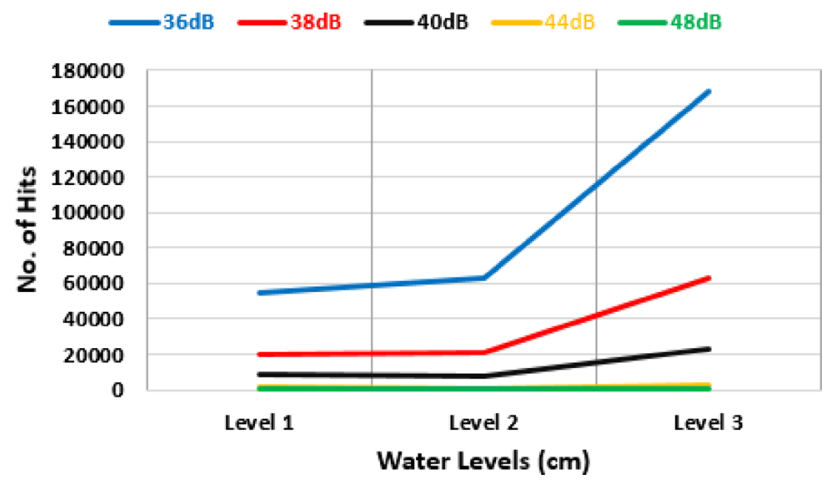

Fig. 7 Effect of threshold levels on the AE signals for channel 1 in tap water

The value of AE signal decreases slowly between 50 and $80{ }^{\circ} \mathrm{C}$ for a power-supply of $2.5 \mathrm{~kW}$. For a power-supply of $3 \mathrm{~kW}$, the level of $\mathrm{AE}$ signal decreases gradually between 50 and $90{ }^{\circ} \mathrm{C}$. It was observed that the powersupply has a significant effect on AE levels and bubble formation behaviour, where the bubbles start to detach from the heating surface at an early stage with a powersupply of $3 \mathrm{~kW}$ compared with the bubble detachment with a power-supply of $2.5 \mathrm{~kW}$. This is because more heat flux and energy are obtained from a $3 \mathrm{~kW}$ power-supply.

\section{Conclusion}

This paper focuses on monitoring and diagnosis of bubble occurrence in boiling processes using AE technology and depends on previous analysis and its investigation. It was observed that AE parameters, such as AE-RMS and threshold levels, are sensitive, robust and reliable in the monitoring and detection of bubble activity and the movement towards the surface of the water. It was observed that the presence of bubble formation in pool boiling is detectable with the $\mathrm{AE}$ technique by applying standard analysis techniques, such as RMS, hits and

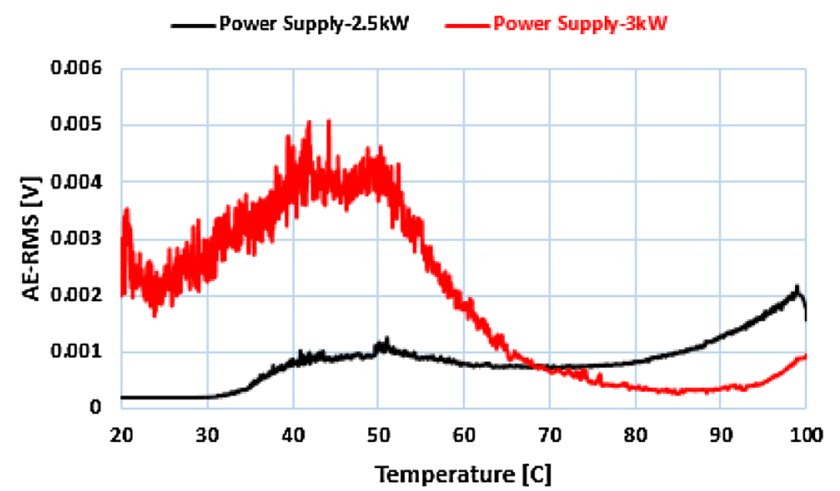

Fig. 8 Effect of power-supply on the AE levels at $20 \mathrm{~cm}$ for channel threshold levels. Furthermore, there is a relationship between bubble formation and temperature during the boiling process. The monitoring of bubble formation using AE technology can complement other condition monitoring methods, all of which are aimed at reducing energy losses and improving life cycle costs. AE technology is capable of detecting the dynamic formation and bursting of bubbles. This technique is necessary for predicting the incidence of boiling in nuclear reactors. This is also the case for observing heat exchanger systems in industrial chemical processes, detecting bubble occurrence in the petrochemical industry, detecting gas layers in oil drilling, and monitoring and detection of cavitation phenomena in centrifugal pumps and valves. It was concluded that the different liquid types have an influence on AE energy levels and bubble formation. In addition, the power supply has a significant effect on AE levels; as the power-supply increases, the levels of $\mathrm{AE}$ signals increase during the bubble formation process.

Finally, this paper provides an early investigation of ongoing research in the application of the $\mathrm{AE}$ technique to detect bubble formation in pool boiling. The authors are also working on another study of bubble formation and collapse in valves that will be published in the near future. Expectantly, the following investigation will contribute to distinguishing the differences between bubble formation and bubble collapse due to cavitation using the AE technique. It is aimed to use waveform analysis for this approach.

Open Access This article is distributed under the terms of the Creative Commons Attribution 4.0 International License (http://crea tivecommons.org/licenses/by/4.0/), which permits unrestricted use, distribution, and reproduction in any medium, provided you give appropriate credit to the original author(s) and the source, provide a link to the Creative Commons license, and indicate if changes were made.

\section{References}

1. Neill, G.D., Reuben, R.L., Sandford, P.M.: Detection of incipient cavitation in pumps using acoustic emission. J. Process Mech. Eng. 211(4), 267-277 (1979)

2. Rydberg, K.E.: Energy efficient water hydraulic systems. In: The fifth international conference on fluid power transmission and control, HangZhou, China, 2001, pp 44-46

3. Weninger, K.R., Camara, C.G., Putterman, S.J.: Energy focusing in a converging fluid flow: implications for sonoluminescence. Phys. Rev. Lett. 83(10), 2081-2084 (1999)

4. Naveen N. Sinha, Los Alamos N. Characterization of liquids using gas bubbles. United States (12), Patent Application Publication (10). 2004; 1(19). http://file:///C:/Users/s218245/Downloads/US20040144175.pdf

5. Brennen, C.E.: Cavitation and bubble dynamics, pp. 145-185. Oxford University Press, Oxford, (1995). doi:10.1017/ CBO9781107338760 
6. Jazi, A.M., Rahimzadeh, H.: Waveform analysis of cavitation in a globe valve. Ultrasonics. 49(6-7), 577-582 (2009). doi:10.1016/j. ultras.2009.02.004

7. Brennen, C.: Hydrodynamics of Pumps. NREC, Oxford University Press (1994). doi:10.1017/CBO9780511976728

8. Alssayh, M.A.: Slug velocity measurement and flow regime recognition using acoustic emission technology. PHD Thesis. Cranfield University; 2013

9. FDIS ISO. International Standards Organization Documents 22096. Documents. ISO, Generic, R.T. (eds.) Condition monitoring and diagnosis of machines. Acoust Emiss; 2007

10. Alhashan, T., Elforjani, M., Addali, A., Teixeira, J.: Monitoring of bubble formation during the boiling process using acoustic emission signals. Int J Eng Res Sci. 2(4), 66-72 (2016)

11. Benes, P., Uher, M.: Identification of liquid boiling by acoustic emission. Fundam Appl Metrol. 1, 1396-1401 (2009)

12. Carmi, R., Bussiba, A., AI and HI. Detection of transient zones during water boiling by acoustic emission. Acoust Emiss. 2011; 29: 89-97

13. Alhashan, T., Addali, A.: The effect of salt water on bubble formation during pool boiling using acoustic emission technique. 13(5), 51-56 (2016). doi:10.9790/1684-1305085156

14. Addali, A.: Monitoring gas void fraction in two-phase flow with acoustic emission. PHD Thesis. Cranfield University; 2010

15. Husin, S., Mba, D.: Acoustic emission of a single bubble activities. In: Proceedings of the World Congress on Engineering 2010 Vol. II. London UK; 2010. pp. 0-5

16. Jaubert, L., Maillard, S., Sulis, V.: Monitoring of fluidic systems by acoustic emission. In: European conf. AE Testing. France; 2008. pp. 334-339

17. Husin, S.: An experimental investigation into the correlation between Acoustic Emission (AE) and bubble dynamics. Cranfield University (2011). ISBN: 9789881821072
18. Leighton, T.G., Walton, A.J.: An experimental study of the sound emitted from gas bubbles in a liquid. Eur. J. Phys. 8, 98-104 (1987)

19. Minnaert, M.: On musical air-bubbles and the sounds of running water. Philos. Mag. 16, 235-248 (1933)

20. Pumphrey, H. C., Crum, L.: Sources of ambient noise in the ocean and experimental investigation. $\mathrm{PhD}$ thesis. University of Mississippi (1989)

21. Leighton, T.G.: The acoustic bubble: Oceanic bubble acoustics and ultrasonic cleaning. In: Proceedings of Meetings on Acoustics. 2015 ; 24: 1-5. doi:10.1121/2.0000121

22. Strasberg, M.: Gas bubbles as sources of sound in liquids. J. Acoust. Soc. Am. 28(1), 20-26 (1956)

23. Plesset, M. S.: Shockwaves from cavity collapse. Philosophical Transactions of the Royal Society of London, vol. 260, pp. 241-244. Royal Society (1966)

24. Strasberg, M.: Gas bubbles as source of sound in liquids. J. Acoust. Soc. Am. 28, 20-26 (1956)

25. Husin, S., Addali, A., Mba, D.: Feasibility study on the use of the acoustic emission technology for monitoring flow patterns in two phase flow. Flow Measurement Instrum. 33, 251-256 (2013). doi:10.1016/j.flowmeasinst.2013.07.011

26. Lauterborn, W., Ohl, CD.: Cavitation bubble dynamics. Basic. Eng. Am. Soc. Mech. Eng. 4(2),65-75 (1997). doi:10.1016/ S1350-4177(97)00009-6

27. Derakhshan, J.O., Houghton, R.R.J.K.: Cavitation monitoring of hydro turbines with RMS acoustic emission measurements. Proc. World Meet. On Acoust. Emiss. 15, 305 (1989)

28. Blanchard, D.C., Woodcock, A.H.: Bubble formation in modification in the sea and its meteorological signifcance. Tellus $\mathbf{9}$, 145-158 (1957)

29. Ceccio, S., Gowing, S., Shen, Y.T.: The effects of salt water on bubble cavitation. J. Fluids Eng. 119, 155-163 (1997) 